Chapter 14

\title{
Advances in Optical Fiber Laser Micromachining for Sensors Development
}

\author{
João M. P. Coelho, Marta Nespereira, Catarina Silva, \\ Dionísio Pereira and José Rebordão
}

Additional information is available at the end of the chapter

http://dx.doi.org/10.5772/52745

\section{Introduction}

Both lasers and optical fibers technology appeared in the 1960s, being, from the start, close related. Even though the latter gained increased visibility in telecommunications, first experiments using optical fiber sensors are reported from early 1970s. From then on, research in optical fiber sensors has increased taking advantage of their potential when comparing with "traditional" sensors. Although there are many well established techniques to manufacture optical fiber sensors, the use of laser technology as increased as their cost diminishes (at least for older, well matured laser sources technology) and new laser sources appeared. This new tool has the advantage of producing well controlled light beams.

Nowadays, laser processing of optical fibers in the production of fiber-based sensors is an important research theme. In particular, the use of infrared radiation has directed attention as new applications were found and new short pulsed laser technology have been developed. In this chapter we will describe the main technology used and the physical principles involved. The key parameters in laser radiation interaction with the fiber materials will be described as well as the most common types of fiber-based sensors that can be produced. The application of ultraviolet (UV), near-infrared (NIR) and mid-infrared (MIR) radiation in the fabrication of fiber grating(FG) sensors is analysed. The physical principles are described and a comparison between theoretical modelling and experimental results is presented for MIR radiation writing of long-period fiber sensors (LPFG). Micromachining with nanosecond (ns) pulsed near-infrared laser radiation is presented and illustrate an ongoing research in the use of this type of laser to produce new cavity-based optical sensors. Experimental work is presented and its potential application is analysed. 


\section{Laser interaction with optical fiber materials}

Laser interaction with the materials in general and optical fiber material in particular, depends on several parameters. These are related with the laser source (its wavelength and emission regime, mainly) and also on the characteristics of the material itself.

Generally speaking, the common fibers used as sensors are made of glass materials. Although plastic and polymeric materials are also used, usually sensors are produced from fibers made of ultra pure chemicals like silicon tetrachloride $\left(\mathrm{SiCl}_{4}\right)$, germanium tetrachloride $\left(\mathrm{GeCl}_{4}\right)$ and also phosphorus oxychloride $\left(\mathrm{POCl}_{3}\right)$. The improvement of their optical properties is accomplished by doping with germanium, erbium and ytterbium among other rare earths. Nevertheless, in the purpose of this chapter, fused silica (pure or doped) will be considered as the typical bulk material for laser interaction regarding fiberbased optical sensors.

The most common lasers emit either in the UV, visible or infrared (IR). However, UV and IR lasers have been the major players in the field of processing optical fibers given that the resulting interaction mechanisms are more efficient for these wavelengths taking advantage of higher absorption in those regimes.

The two main regimes as laser sources concerns are continuous wave $(\mathrm{CW})$ and pulsed emission. Recent year's laser developments allowed laser sources to present a broad range of available pulsed regimes, from milliseconds (ms) to femtoseconds (fs) pulse widths. This availability has potentiated new ways of using the laser as a tool for optical fiber processing.

Under laser irradiation, and depending on the mentioned source parameters, the main physical mechanisms can be divided in thermal and photonic (non-thermal) effects. These physical processes are used to create different fiber-based sensors, as it will be described in the following sections.

\subsection{Thermal effects}

Thermal processes arise from absorption of the laser energy in the material, and in general apply for continuous wave (CW) operation, long pulse lengths and high-pulse-repetitionfrequency pulse trains. In this case, the absorbed radiation creates an excess of energy due to the excitation of the lattice which is transformed into heat, increasing the material's temperature from its surface to its bulk by heat conduction, so the most basic thermal effect is heating, that depends on irradiation time and thermal diffusivity of the material.

Heating is the effect behind LPFG fabrication using $\mathrm{CO}_{2}$ laser, where the refraction index change is achieved by heating a fiber submitted to a tensile stress. If the irradiance is high enough, phase transformations are produced. For silica-base materials, melting is produced when the irradiance has a magnitude of $\sim 10^{5} \mathrm{~W} / \mathrm{cm}^{2}$ and depending on the irradiation time, the melted material increases its depth into the bulk. Once the boiling point is achieved, if the irradiance reaches values of $>\left(10^{5}-10^{8}\right) \mathrm{W} / \mathrm{cm}^{2}[1]$ vaporization is initiated. This last step is the basis of the thermal photoablation, which consists in the precise removal of material, by surface vaporization or spallation (due to thermal stresses) [1]. 


\subsection{Photonic effects}

Photoionization is a type of laser matter interaction by which a laser pulse modifies the fundamental structure of a material through physical processes like: non- thermal excitation, ionization and dissociation of atoms and molecules, depending on the light and material properties. The simplest process is the single photon ionization (SPI) consisting in the absorption of a single photon with resulting removal of one electron. This process is strongly dependent on the wavelength, laying in the UV for the interaction with glass materials, and requires low irradiance levels $\left(<10^{7} \mathrm{Wcm}^{-2}\right)$ [1]. This effect is the basis of the laser induced refraction index changes in FG fabrication where this kind of photochemical reaction is produced on UV photosensitive Ge-doped fibers.

The mechanisms of photosensitivity can be explained by the interaction of UV radiation in a special structure in the fiber's bulk named Germanium oxygen deficient center (GODC), which is able to absorb one or two photon. The photosensitivity mechanism is intrinsically associated with the dopants incorporated during the silica-based optical fiber fabrication. Therefore, it is plausible that the origins of this process are related with the germanosilicate glass synthesis, in which a controlled sequence of chemical reactions that involves a mixture of several gases at high temperature occurs accordingly with the reactions [2]:

$$
\left\{\begin{array}{l}
\mathrm{SiCl}_{4}+\mathrm{O}_{2} \stackrel{\mathrm{T}}{\rightarrow} \mathrm{SiO}_{2}+2 \mathrm{Cl}_{2} \\
\mathrm{GeCl}_{4}+\mathrm{O}_{2} \stackrel{\mathrm{T}}{\rightarrow} \mathrm{GeO}_{2}+2 \mathrm{Cl}_{2} \\
\stackrel{\mathrm{T}}{\rightarrow} \mathrm{GeO}+\mathrm{O}
\end{array}\right.
$$

These reactions show that the presence of germanium promotes $\mathrm{GeO}$ formation. Truly, the formation of $\mathrm{GeO}$ defects is promoted due to the thermodynamics of the gaseous germanium redox reaction at the high synthesis temperature and is dominant, since the Ge-O bond is weaker than the $\mathrm{Si}-\mathrm{O}$ bond. Despite the possibility of other suboxides being formed, $\mathrm{GeOx}$ $\{x=1$ to 4$\})$, the $\mathrm{GeO}$ is the most common sub-product inside the germanosilicate glass amorphous structure, $\mathrm{GeO}_{2}-\mathrm{SiO}_{2}$, as a source of glass defects [3]. The GODC, occurs when a $\mathrm{Ge}$ atom is bonded to a $\mathrm{Si}$ or Ge atom, in the absence of an oxygen atom, giving rise to a strong absorption at $242 \mathrm{~nm}$ band [3]. The model of an oxygen vacancy neighbouring a Ge atom was suggested, based on the analogy of the spectroscopic properties of this Ge-related defect with those monitored on an oxygen vacancy in pure $\mathrm{v}-\mathrm{SiO}_{2}$. This is consistent with the one photon nature pathway, corresponding to the GODC's triplet state andits intensity increases linearly with the concentration of $\mathrm{GeO}_{2}[4]$.

The photosensitivity mechanism can also be triggered through a two photon absorption mechanism, and its efficiency is affected by several parameters like light's power density, attenuation and light [5]. Despite the fact that pure silica glasses exhibits poor photosensitivity to UV-laser light even if exposed to large accumulated fluence values close to $100 \mathrm{~kJ} / \mathrm{cm}^{2}$, 
this can be reversed when a fs-laser beam at $\approx 800 \mathrm{~nm}$ wavelength is used [6]. In this case, strong permanent changes in the refractive index $\left(2-6 \times 10^{-3}\right)$ are attainable.

The two photon absorption phenomenon is considered one of the multi-photon ionization (MPI) processes which consist in the absorption of two, three or even five photons exciting the electrons to the conduction band. The difference between the two processes can be explained comparing the number of photoproducts versus the irradiation intensity [7]. Typically, this is a high-intensity $\left(\mathrm{I} \sim 10^{11}-10^{13} \mathrm{Wcm}^{-2}\right)$ [7] and very fast process, lying in the fsrange. Two regimes are distinguished, fs-UV and fs-IR, according with the wavelength employed. In the UV regime, the main mechanism is the previously two photon absorption while in the IR mechanism the three and five photon absorption are predominant.

Laser-induced optical breakdown is a process of photoionization which has the result of plasma formation and photoablation. The main photoionization mechanisms are the already mentioned SPI and MPI. For ps- and ns-pulses the optical breakdown is explained by the avalanche model. It's a damage mechanism that starts with one or more electrons in the conduction band, heated by the laser field. The electron collides with the matrix, gaining enough kinetic energy (by inverse Bremstrahlung) to free a second electron. The same process repeats until the electron density approaches the critical plasma density $\sim 10^{9} \mathrm{e}^{-} / \mu \mathrm{m}^{3}$, resulting in photoablation. An inconvenient is that in the ns-time scale, most of the plasma energy is transferred to the matrix being able to produce collateral thermal damage and fractures, worsening the quality of ablation [8]. This effect can be avoided in the fs-scale, since there's no time for an avalanche fully develop, and MPI assumes equal importance to electron avalanche. Thus, the heat diffusion is frozen and thermal damages are eliminated. This process is known as "cold ablation" [8].

Theoretically, according to the electron avalanche model, the laser fluence threshold for ablation is strongly dependent to the laser wavelength, implying that this threshold should increase slightly as the wavelength decreases but reported experimental data shows the opposite. This could mean that other photoionization processes could be implied in optical breakdown of silica and having in mind that lattice defects are more absorptive in the UV than in MIR [8].

\section{Fundamentals of optical fiber sensors}

The understanding of the potential of using laser technology to create fiber-based sensors depends also on the understanding of the requirements those sensors have. The process of interaction must lead to a certain change in the fiber properties that must produce the required sensitivity to an external change. In this section, the fundamentals of the most common fiber-based sensors is presented with focus on those being targeted as able to be produced by laser irradiation. Cavity-based sensors and refractive-index modulated sensors principles will be described. 


\subsection{Optical fiber grating sensors}

FGs are optical devices based in the principle of photo-refractive effect first discovered by Hill et al. [9]. Since then, their development had a significant impact on research and development of telecommunications systems and fiber optic sensors. It use as sensing element is advantageous due to the intrinsic characteristics of the fiber sensors, such as multiplexing, remote sensing, high flexibility, low propagating loss, high sensitivity, low fabrication cost, weight and compactness, high accuracy, simultaneous sensing ability, and immunity to electromagnetic interference.

FGs are often classified into two types: Bragg gratings (also called reflection or short-period gratings), in which coupling occurs between modes travelling in opposite directions; and transmission gratings (or LPFGs), in which the coupling is between modes travelling in the same direction. These optical devices are comparatively simple and in its most basic form, it consists on a periodic modulation of the properties of an optical fiber (usually the refraction index of the core). This can be made by permanent modification of the refractive index of the optical fiber core or by the physical deformation of the fibre. In this section, it is presented the fundamental aspects of both types of gratings, and their sensing application.

\subsubsection{Fiber Bragg grating}

FBGs are spectral filters based on the principle of Bragg reflection. These periodic structures operate in reflection mode and are manufactured with a period of less than $1 \mu \mathrm{m}$. Their submicron period provide coupling between the modes that propagate in opposite directions. The principle of operation of these optical devices is schematized in Figure 1. A standard FBG consists of a refractive index modulation in the core of an optical fibre that acts to couple the fundamental forward propagating mode to the contra-propagating core mode. When a broad-spectrum light beam inside in the fiber grating, a narrow wavelength range is reflected and all other wavelengths are transmitted. The reflected light signal will be centered at the Bragg wavelength. The spectral response of the FBG is governed by the phase matching condition, $\lambda_{B}=2 n_{\text {eff }} \Lambda$, where $\lambda_{B}$ is the Bragg wavelength, $n_{\text {eff }}$ the effective refractive index of the fiber core and $\Lambda$ the Bragg grating period [10]. Any change in the modal index or grating pitch of the fiber caused by strain or temperature results in a shift of the Bragg wavelength.



Figure 1. Schematic representation of Fiber Bragg grating principle of operation. 
Consider a uniform Bragg grating formed within the core of an optical fiber. The refraction index profile can be expressed as $n(x)=\Delta n \cdot \cos (2 \pi / \Lambda)$, where $\Delta n$ is the amplitude of the induced refractive-index perturbation (typically, $10^{-5}-10^{-2}$ ) and $x$ is the distance along the fiber's longitudinal axis. The coupled-mode theory analytical enables the description of the reflection properties of Bragg gratings. The reflectivity of a grating with length $L$ and constant modulation amplitude and period is given by $R(L, \kappa)=\tanh ^{2}(\kappa . L)$ [11] were the coupling coefficient for a single mode fiber is $\kappa=\pi . \Delta n / \lambda$.

FBGs have been applied in telecommunications[12] and also for a wide variety of sensing applications in several fields [12]. However, FBGs has practical implementation limitations, including the needs of special post-processing for sensing of external refractive index and reduction of the sensor's mechanical strength [13].

\subsubsection{Long period fiber gratings}

LPFGs are produced by inducing a periodic refractive index modulation (tipically $10^{-4}$ ) in the fiber core with periods typically in the range from $100 \mu \mathrm{m}$ to $1000 \mu \mathrm{m}$ [14]. These optical devices operate in transmission mode and their large modulation period promotes the light coupling between co-propagating modes of the optical fibre. In the case of single mode fibers, this takes place between the fundamental and cladding modes, in the same direction. This principle is illustrated in Figure 2. The cladding modes are quickly attenuated resulting in a series of attenuation bands in the transmission spectrum. Each attenuation band corresponds to coupling to a different cladding mode. The phase matching wavelengths are governed by the expression $\lambda_{\text {res }}{ }^{m}=\left(n_{\text {eff,co }}-n_{\text {eff,cl }}{ }^{m}\right) . \Lambda[10,15]$, where $\Lambda$ is the grating period, $n_{\text {eff,co }}$ and $n_{\text {eff }, c l}$ are the effective refractive indexes of the core and $m$ th-cladding modes, respectively. The refractive index sensitivity of LPFGs arises from the dependence of the coupling wavelength upon the effective index of the cladding mode.

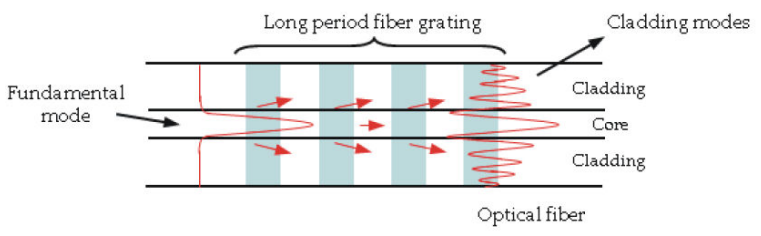

Figure 2. Schematic diagram of long period fiber grating.

Light transmission through the core follows a sinusoidal function of the core refractive index modulation for the wavelengths in the resonance [16] is given by $T=\cos (D . L / 2)$, where $L$ is the grating length and $D$ is a coupling coefficient proportional to the core index modulation. The bandwidth of the resonance dips depends on both the coupling coefficient and the difference between the core and cladding indexes: 


$$
\Delta \lambda_{\text {FWHM }}=\frac{\lambda_{m}^{2}}{\left(n_{\text {eff }, c o}-n_{e f f, c l}^{m}\right)} \sqrt{\frac{4 D}{\pi L}}
$$

External changes in parameters like refractive index, temperature or strain can affect the terms in the ruling equations and consequently shift the attenuation dips and alter their bandwidths [14]).

These optical devices are very sensitive to changes in physical parameters, such as, temperature, strain, bending, torsion, and refractive index of the surrounding medium [17]. This makes possible the use of the LPFGs as a multi-parameter sensor [16]. Their sensibility of to external environment parameters is determined by the magnitude of the perturbation in the refractive index, the period of the grating, and its length.

Compared to other optical devices, LPFGs have a number of unique advantages such as low insertion losses polarization independence, high temperature sensitivity, and relatively simple fabrication. A further advantage of these devices is their higher sensitivity to the environmental refractive index change without the need for access to the evanescent field, as in the case of the FBGs. The extreme sensitivity of the LPFGs to environmental changes could be a disadvantage in telecommunications devices (cross sensitive problems).

\subsection{Cavity based optical fiber sensor}

Optical fiber Fabry-Perot (FP) interferometric sensors are the main cavity-based type of fiber-sensors and demonstrate a great versatility in different applications $[18,19]$. The cavitybased sensors are particularly attractive due to its inherent advantages, including small size, relatively low temperature cross-sensitivity and corrosion resistance, high sensitivity, high frequency response and immunity to electromagnetic interference.

In its simplest form, the FP cavity consists in two reflective surfaces arranged in parallel forming a resonant cavity. The reflections at the two end surfaces of the cavity create an interference signal which is a function of the length and refractive index of the cavity. Changes in environment causes a phase shift in the interference pattern and, as a result, a fiber FP sensor is capable of measuring various parameters including temperature, pressure, strain [20]. Considering a general analysis, the transmission function is [13]:

$$
T=\frac{\left(1-R^{2}\right)}{1-R^{2}-2 R \cos (\phi)}
$$

being $R$ the reflectivity of the surfaces, assuming that both are equal, and that the phase difference between each succeeding reflections is $\varphi=4 \pi$.n.L. $\cos (\theta) / \lambda$.

The principle of a sensor based in devices like these is based in the fact that changes on the cavity distance (or angle) or in the refractive index of the different media produces a change 
in the transmitted signal (or reflected, since, the reflected signal is equal to $1-T$, not considering absorption). The resolution of the sensor can be evaluated through a parameter named finesse, $F$, relating the distance between peaks, $\Delta \lambda$, and the full-width half-maximum of the peaks, $\delta \lambda: F=\Delta \lambda / \delta \lambda$. Naturally, real fiber sensors are more complex and the applied theory differs from case to case.

Traditionally, FP cavities have been divided in intrinsic (where the sensing element is the fiber itself), extrinsic (two fiber pieces physically separated forming a cavity bounded typically with a capillary glass tube) or hybrid (splicing sections of different types of fibers, for example)[18,21-23]. However, other methods of creating these cavities have been researched like chemical etching and laser processing.

Chemical etching is an efficient and low cost way of producing FP cavities in optical fibers, but the control of the cavity length is less accurate and depends on the precise control of the process, mainly the duration of the etching[24].

Recent methods use laser beams to produce the cavities, either by removing material laterally in the fiber [25] or opening holes on the fiber's end [26] as schematized in Figure 3. Ran et al. present an interesting example [26] of a refractive index sensor based on a cavity created by a $157 \mathrm{~nm}$ wavelength laser beam on the end of an optical fiber. The micropatterned fiber is then spliced to another fibercreating an air cavity. With this geometry (Figure 3(a)), the refractive index measures can be accomplished without the need of filling the micrometric cavity. In this case, reflection in a third interface must be considered and equation (1) replaced accordingly $[20,26]$, and the analyzed signal is the reflected instead of the transmitted. This type of sensor, with a cavity formed from a hole with a depth of around $20 \mu \mathrm{m}$ and $56 \mu \mathrm{m}$ diameter (and $1 \mathrm{~mm}$ distance to the tip), allowed to measure refractive indexes of liquids with a resolution of $\sim 4 \times 10^{-5}$, and is considered as the guideline for the research presented in section 5 .

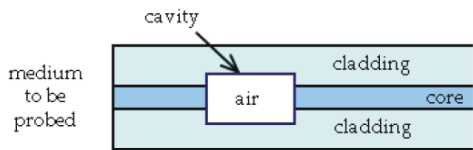

(a)

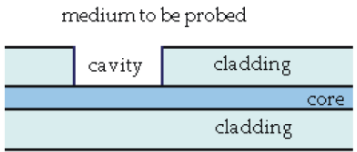

(b)

Figure 3. Schematic of two possible configurations for cavity-based optical sensors.

\section{FGs sensors fabrication using laser radiation}

FGs are important fiber-based sensors. Traditionally they are produced by arc-discharges or UV-exposure. However, in the last years the use of $\mathrm{CO}_{2}$ lasers, emitting in the MIR, and fs lasers, emitting in the NIR, to write FGs has emerged as an important alternative. In this section, the main laser manufacturing techniques of fiber grating sensors are presented, consid- 
ering UV, MIR and NIR radiations. In this scope, an analytical theoretical model for the writing of LPFG by MIR radiation is presented and compared with experimental data.

\subsection{FG writing using UV lasers}

The use of UV laser radiation was in the base of both FBG and LPFG development. The formation of gratings in an optical fiber was first reported in 1978 by Kawasaki et al. [27] using an argon-ion laser at $488 \mathrm{~nm}$ UV wavelength. A few years latter, the first LPFG was introduced in 1995 by Vengsarkar who exposed photosensitive optical fibers to 242-248 nm wavelength UV krypton fluoride, $\mathrm{KrF}$, laser light [28].

Although the first FBGs have been manufactured by internal writing [27] (using the interference between the transmitted beam and reflected beams) and holography (two overlapping UV light beams interfere producing a periodic interference pattern) [29], the phase-mask technique has quickly become usual, and even used (in a similar way) from the start for LPFG writing.

Usually, the phase-mask is made from a flat piece of silica glass (transparent to UV radiation) where a one dimensional periodic surface relief is etched (using photolithographic techniques) in one of the surfaces. Thus, the phase-mask becomes an optical element with the capability to diffract the UV beam in transmission. The interference of the transmitted beams corresponds to different diffraction orders in the proximity of the surface, originating a fringe pattern, and leading to Bragg gratings fabrication by modulation of the refractive index in the core of the optical fibre. The profile of the phase grating is chosen such that the zero-order diffracted beam is suppressed to less than $1 \%$ of the transmitted power. In addition, the principal beams diffracted by the phase-mask correspond to plus and minus first orders, containing each one, typically, more than $35 \%$ of the transmitted power. Then the produced interference pattern photo-imprints a refractive index modulation in the core of the photosensitive optical fibre placed in contact, or in close proximity, immediately behind the phase mask. Typically, the fringe pattern is focused along the fiber's core with the help of a cylindrical lens. The phase-mask technique has the advantage of greatly simplifying the manufacturing process for Bragg gratings, yet yielding high performance gratings. In comparison with the holographic technique, the phase-mask technique offers easier fiber/laser alignment, reduced stability requirements on the writing apparatus and lower coherence requirements on the UV laser beam.

Another writing method uses the point-by-point technique. In this case, single UV laser beam is used to imprint the grating into the fibers equentially along the fiber's length. The incident laser beam is focused on the optical fiber core or cladding (for either FBG or LPFG, respectively) using a lens. The periodic irradiation is accomplished by computer control of the laser beam and the movement of the fiber, so the periods are inscribed. Another way to produce the periodic inscription is by scanning the laser beam focus over the optical fiber, not only to produce the longitudinal modulation but also to produce each transversal refractive index change zone. This process is illustrated in section 4.2.2 regarding MIR irradiation techniques. Figure 4 illustrate both writing techniques. 
The mentioned methods apply independently of the UV laser used, and thus from the physical mechanisms involved (see section 2). However, laser technology significantly differs, a characteristic of producing FG using UV laser radiation. Usually, excimer lasers are used to write FGs through the single- or double-photon low energy physical principles described in section 2 . Wavelengths of $488 \mathrm{~nm}$, and in the ranges $333 \mathrm{~nm}$ to $364 \mathrm{~nm}$ or $244 \mathrm{~nm}$ to $288 \mathrm{~nm}$ are typical either for FBGs or LPFGs. Besides applied wavelength, the required irradiances depend strongly in the optical fiber being considered (mainly its photosensitivity characteristics) but can roughly being considered in the range from a few $\mathrm{W} / \mathrm{cm}^{2}$ to tenths of $\mathrm{MW} / \mathrm{cm}^{2}$ [7].

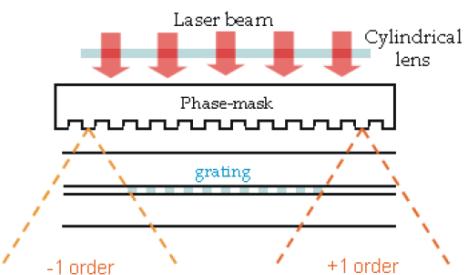

(a)

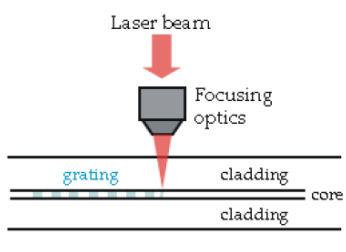

(b)

Figure 4. Illustration of (a) phase-mask and (b) point-by-point writing techniques

Regarding multiphotonic high-excitation energy UV irradiation, this is accomplished using the (relatively) new fs-pulsed laser technology, typically emitting with wavelengths lower than $248 \mathrm{~nm}$. In these cases, irradiances are in the order of $\mathrm{GW} / \mathrm{cm}^{2}$ or higher [7]. This technology based in fs-pulses allows obtaining excellent quality FGs mainly to the laser high spatial uniformity [7]. However, this technology is still very expensive which limits its broader use when comparing with other technologies (either in UV or IR).

\section{2. $\mathrm{LPFG}$ writing using $\mathrm{CO}_{2}$ lasers}

The use of $\mathrm{CO}_{2}$ lasers to produce LPFGs was first reported by Davis et al. [30] and Akiyama et al. [31] in 1998. From then on, the application of this technology has lead to an increasing research on its application for the development of new optical fiber sensors [32].

Using this type of MIR emission laser has several advantages regarding the other two well established methods (UV lasers and arc discharges). The gratings can be inscribed directly in most telecommunication fibers, support high temperatures without vanishing (in opposition to those produced by UV) the process has high repeatability and predictability (in opposition to the arc-discharge method). Also, since $\mathrm{CO}_{2}$ laser systems are commonly used to process several materials and have a long established industrial application, available systems are robust and low-price.

The application of MIR laser radiation to produce a LPFG has physical principles similar to the ones considered for arc-induced LPFGs [33]. Both rely in thermal effects acting in the fiber bulk materials. However, while the latter can be considered as a volume effect, being applied along the transversal section of the fiber, between the two electrodes, the material's 
high superficial absorption considered in MIR irradiation promotes heat conduction as a major player in the physical mechanisms involved.

\subsubsection{Physical mechanisms}

Considering a standard silica-based optical fiber under tension and irradiated by a (Gaussian) $10.6 \mu \mathrm{m}$ wavelength beam emitted from a $\mathrm{CO}_{2}$ laser, two main phenomena must be considered: the thermal heating due to the interaction between the photons and the glass molecular structure and the stress due to the differences between a relatively low-viscosity doped silica core and a relatively high-viscosity pure silica cladding [34]. Differences between core and cladding thermal expansion coefficients and viscosity lead to residual thermal stresses and draw-induced residual stresses. These effects are localized and, when periodically induced in the fiber's length, can be responsible for the creation of the gratings. This effect is due to the refractive index change resulting from frozen-in viscoelasticity [35].

The temperature distribution $T(r, z, t)$, with $r^{2}=x^{2}+y^{2}$ for laser heating of a homogeneous medium can be obtained by solving the $2 \mathrm{D}$ heat flow equation. Considering $K=K(T)$, defining the thermal diffusivity $k\left[\mathrm{~m}^{2} \mathrm{~s}^{-1}\right]$ as $k=K /(\rho C p)$, where $\rho$ is the density, $C_{p}$ the specific heat, $K$ the thermal conductivity and assuming them constants, the resulting temperature can be approximated for Gaussian elliptical laser beams through [36-40]:

$$
\begin{aligned}
T(x, y, z, t)= & \frac{(1-R) P}{4 \pi k w_{x} w_{y}} \int_{0}^{\sqrt{K t}} \Psi(x, y, s) \cdot\left[\exp \left(a_{T} z\right) \operatorname{erfc}\left(\frac{a_{T} S}{2}+\frac{z}{s}\right)+\right. \\
& \left.+\exp \left(-a_{T} z\right) \cdot \sqrt{a^{2}+b^{2}} \cdot \operatorname{erfc}\left(\frac{a_{T} S}{2}-\frac{z}{s}\right)\right] d s
\end{aligned}
$$

with

$$
\Psi(x, y, s)=\frac{a_{T} s}{\frac{s^{2}}{\left(w_{x} w_{y}\right)}+1} \cdot \exp \left[\frac{x^{2}}{w_{x}^{2}+s^{2}}-\frac{y^{2}}{w_{y}^{2}+s^{2}}+\frac{\left(a_{T} s\right)^{2}}{4}\right]
$$

being $R$ the reflectivity at the air/fiber interface for the assumed wavelength, $P$ the laser power, $a_{T}$ the absorption coefficient (assumed constant) and $w_{x}$ and $w_{y}$ the beam's radii at focus (for each axis). With the temperature, $T$, the resulting residual thermal stresses can be calculated using [37]

$$
\sigma_{x}=\frac{E}{1-v}\left[\frac{2 v}{r_{c}^{2}} \int_{r=0}^{r_{c}} \alpha T r d r-\alpha T\right]
$$


being $r_{c}$ is the radius (cladding or core), $E$ is the Young's modulus and $v$ the Poisson's ratio.

If the core is the lower viscosity glass (e.g. Ge-doped silica core with pure silica cladding), the residual axial elastic stresses in the cladding and core, $\sigma_{c l}$ and $\sigma_{c o r}$ respectively, resulting from a draw tension $F$, over the equivalent cross-sectional areas $A_{c l}$ and $A_{c o}$ can be obtained from [37]:

$$
\sigma_{x, c l}=\frac{F}{A_{c l}}\left(\frac{A_{c o} E_{c o}}{A_{c o} E_{c o}+A_{c l} E_{c l}}\right) \quad \text { and } \quad \sigma_{x, c o}=F\left(\frac{E_{c o}}{A_{c o} E_{c o}+A_{c l} E_{c l}}\right)
$$

Taking in consideration the mentioned stresses, the refractive index change in a silica-based optical fiber can be approximated by the relation [35] $\Delta \mathrm{n} \approx-6.35 \times 10^{-6} \sigma$, where $\sigma$ represents the overall (both thermal and drawn-induced) residual stresses (in MPa) in the fiber's axial direction. Accordingly with Yablon [34], stresses in the other directions can be neglected.

Besides stress-related refractive index change, localized heating can induce microdeformation of the fiber and also changes in its glass structure. The later is likely to occur in the core for which the fictive temperature (below the fictive temperature the glass structure doesn't change) is lower $[33,41]$. As an example, it can be found that, for a Ge-doped core, the fictive temperature ranges from $1150 \mathrm{~K}$ and $1500 \mathrm{~K}$ [41].

These analytical equations don't consider all the physical phenomena (e.g. convection and radiation losses) and were developed assuming several simplifications (mainly, neglecting the temperature dependence of the glass parameters). However, their capability of being used as an engineering tool to develop fiber optic sensors has been demonstrated [40]. A detailed analysis can be made using numerical methods and considering that the absorption coefficient is temperature dependent, e.g. accordingly with MacLachan and Meyer [42].

\subsubsection{Irradiation methodologies}

Since there is still no phase mask available for $\mathrm{CO}_{2}$ laser radiation, methodologies rely basically in the point-by-point technique. Nevertheless, several methodologies have been tested since the first experiences in 1998 and are resumed in the schematic of Figure 5. As an example, Davis [30] and Akiyama [31] both have written each single period of a grating by focusingthe laser beam by means of spherical lenses. Spots had dimensions of about $140 \mu$ mand translation stages moved the fiber under the laser spot. They used a CW laser, and the single pulse duration was defined through a computer-controlled shutter.

Usually, $\mathrm{CW} \mathrm{CO}_{2}$ laser technology is chosen due to its availability and cost. Low power lasers and mechanical shutters allowing hundreds of ms pulses perform well and accomplish the required performances. Q-switch $\mathrm{CO}_{2}$ lasers [43] have also been reported by Raoet al. [44]. In this case, shorter pulses are available at high frequency rate (in the order of $\mathrm{kW}$ ). Nevertheless, since fluence is the main parameter involved in the interaction process, setting laser power, pulse duration and spot radius should lead to similar results [40]. 

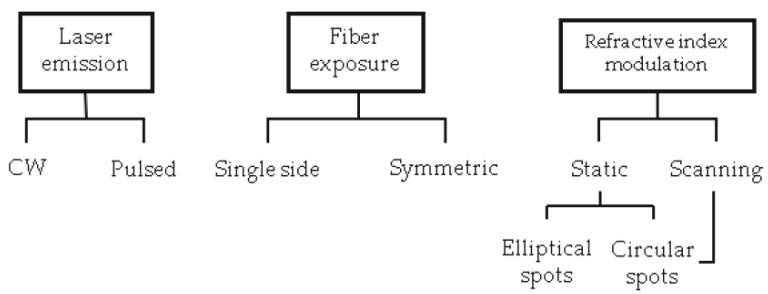

Figure 5. Schematic illustrating the different irradiation methodologies that can be applied for each available operational parameter.

Regarding the way each refractive index modulation is created, there are mainly two options: a static irradiation, for which the laser is applied for a determined amount of time, and a dynamic irradiation where the laser beam is scanned over the region where the refractive index change is to be created. In the first case, basically, one must ensure that the region is fully irradiated (i.e. the focused spot is larger that fiber's diameter) while in the scanning procedure requires the opposite (spot size smaller that the fiber's diameter).

Figure 6 schematizes the two situations considered for the static procedures and the one for scanning. For the latter (Figure 6(a)), the usual procedure is to have the laser beam focused in a small spot and scanned it over the fiber using a galvanometric mirror. If two of such mirrors are used, one of them can be used to move the beam longitudinally and thus write the full LPFG without moving the fiber. However, these scanners and associated optics are expensive, and accomplishing small spots is difficult for the considered wavelength. The diffraction limited spot radius, $w_{\mathrm{d}}$, resulting from focusing an initial beam of wavelength $\lambda$ and radius $w_{0}$ using a lens of focal distance $\mathrm{f}$ is: $w_{\mathrm{d}}=1.22 \lambda . \mathrm{f} / w_{0}$.

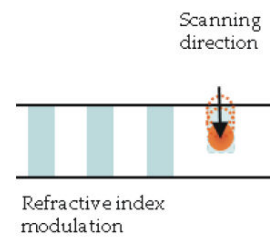

(a)

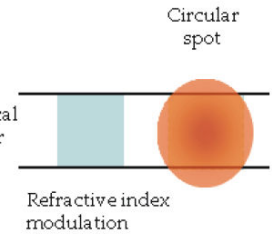

(b)

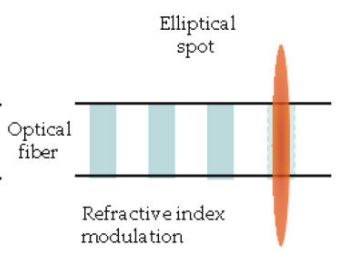

(c)

Figure 6. Illustration of (a) dynamic scanning and static (b) circular and (c) elliptical spots procedures in creating LPFG in an optical fiber.

Figure 7 shows the diffraction limited spot radius values for a $10.6 \mu \mathrm{m}$ wavelength beam focused by different lenses. Two situations are plotted: one considers that the laser has an initial $3.5 \mathrm{~mm}$ radius (a usual value) and the other that this value doubles (e.g. using a $2 \mathrm{x}$ beam expander). Also plotted is the dimension (cladding radius) of a common optical fiber (for the case, the SMF-28, already considered previously). The plot indicates that only for the lowest focal lengths ( $<20 \mathrm{~mm}$, averaging for the two situations) one can obtain spot sizes smaller 
than the optical fiber radius. The common situation is to use focal lengths in the order of 50 $\mathrm{mm}$, and typically spot sizes are in the order of hundreds of microns. This leads to the fact that usually a static approach is used. Since a circular spot creates (potentially) larger affected zones (Figure 6(b)) and, for smaller beams makes it more difficult to align relatively to the fiber, elliptical beams (Figure 6(c)) are often the preferable choice. This is accomplished by using a cylindrical lens with its axis perpendicular to the fiber's axis.

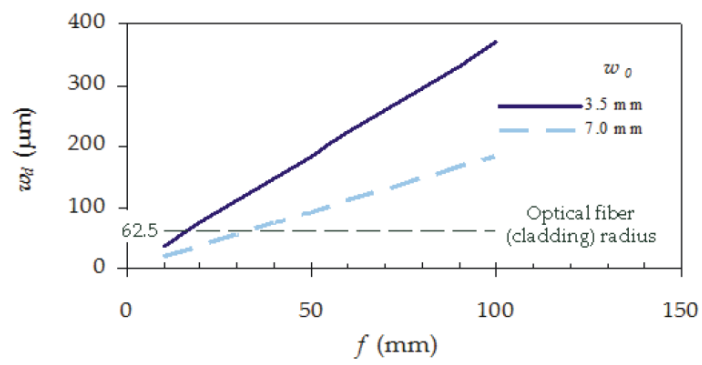

Figure 7. Diffraction limited spots for $w_{0}=3.5 \mathrm{~mm}$ or $w_{0}=7.0 \mathrm{~mm} \mathrm{CO}$ laser beam radius focused by different focal length lenses.

While no major difference in the LPFG performance has been reported regarding the above mentioned different techniques, the single-side and symmetric exposure to the laser radiation were compared by Oh et al. [45], demonstrating that the polarization-dependent loss of the first fabrication method $(1.85 \mathrm{~dB}$ at $1534 \mathrm{~nm})$ could be significantly reduced to $0.21 \mathrm{~dB}$ by applying the second method. Nevertheless, due to its simplicity, the single-side exposure is the most commonly used methodology and the accomplished performance still fulfils the usual requirements.

The same techniques, applied with different parameters (e.g. laser power and applied weight) can produce different devices like based on tapers or grooves along the fiber (i.e., zones were the cladding diameter is reduced) [46]. Other possible advances can be accomplished in the future regarding the writing of non-uniform (or "chirped") LPFG, where the period changes along the grating, and direct writing by MIR interferomety [46].

\subsubsection{An example}

Considering a standard single-mode fiber, SMF-28 [47], consisting of a core of $3.5 \mathrm{~mol} \%$ Gedoped $\mathrm{SiO}_{2}$ and a pure fused silica cladding and irradiating with a common $\mathrm{CO}_{2}$ laser a simple example can illustrate the application of the formulae and also correlate with experimental data. Table 1 presents the fiber's main parameters considered for the calculations and their references. Values from Yang et al. [36] are considered for the $10.6 \mu \mathrm{m}$ wavelength of $\mathrm{a} \mathrm{CO}_{2}$ laser and equals for both the core and the cladding. This assumption can be made mainly since the Ge concentration in the fiber's core is very low $[7,48,49]$. 
Using a static asymmetrical irradiation with a $\mathrm{CW} \mathrm{CO}_{2}$ laser and a cylindrical lens to have a $w_{x}=0.15 \mathrm{~mm}$ and $w_{y}=1.75 \mathrm{~mm}$ elliptical spot on the fiber, the implemented setup is schematized in Figure 8(a) and the considered referential in Figure 8(b). In practice, a Synrad 48-2 laser and a $50 \mathrm{~mm}$ focal length lens were used. The laser operation was computer controlled with emissions in the order of hundreds of ms. Experimental set-up also consisted of a broad band light source (Thorlabs S5FC1005S) and an optical spectrum analyzer (OSA) to monitor the LPFG fabrication, while a fast camera (PCO SensiCAM), perpendicular to the irradiation axis, allows to optically visualize the process. The irradiated zones were analyzed using an optical microscope with amplifications up to $1,000 \times$.

\begin{tabular}{lcc}
\hline Parameter & Core & Cladding \\
\hline Radius, $w(\mu \mathrm{m})[47]$ & 4.1 & 62.5 \\
Refractive índex (@ 1550nm, 300K), $[$ [7] & 1.449 & 1.444 \\
\hline Young's modulus, $E(\mathrm{GPa})[49]$ & 70.8 & 72 \\
\hline Poisson's ratio, $v[49]$ & 0.165 & 0.173 \\
\hline Reflectivity (@ 10.6 $\mu \mathrm{m}), R[36]$ & & 0.15 \\
\hline Density, $\rho\left(\mathrm{kg} / \mathrm{cm}^{3}\right)[36]$ & & $2.2 \times 10^{-3}$ \\
\hline Specific heat, $C_{p}(\mathrm{~J} / \mathrm{kg} \mathrm{K})[36]$ & & 703 \\
\hline Thermal diffusivity, $K\left(\mathrm{~m}^{2} / \mathrm{s}\right)[36]$ & & 2 \\
\hline Absorption coefficient $(@ 300 \mathrm{~K}), a_{T}\left(\mathrm{~cm}^{-1}\right)[36]$ & & 250 \\
\hline
\end{tabular}

Table 1. Optical fiber parameters considered for the calculations.

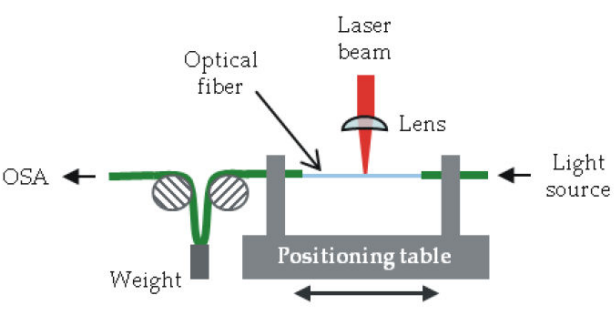

(a)

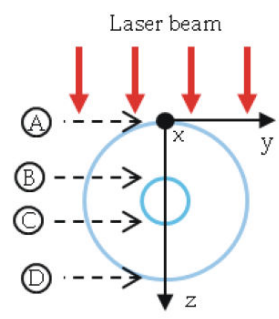

(b)

Figure 8. a) Schematic apparatus of a LPFG writing by laser and (b) optical fiber cross-section indicating the considered referential and the interfaces between the different regions: $A$ - irradiated surface, B - core/cladding interface (upper), C-core/cladding interface (lower) and D - bottom surface.

Figure 9(a) shows a microscope photo of an irradiated fiber, part of a $25 \mathrm{~mm}$ length grating with a period of $500 \mu \mathrm{m}$ and Figure $9(\mathrm{~b})$ the resulting relative transmission spectrum.Besides the general conditions previously mentioned, a weight of $16 \mathrm{~g}$ was applied and a laser 
power of $6 \mathrm{~W}$ was delivered for the duration of $600 \mathrm{~ms}$. In this image it is possible to observe an affected area along the fiber's axis of about $130 \mu \mathrm{m}$. Also visible is a (small) micrometric deformation of the fiber.

Using equation (4), one can obtain the temperature distribution at the different regions illustrated in Figure 8(b). Figure 10 shows this distribution along the fiber's axis as well as the equivalent zone regarding the size of the visible affected zone observed in Figure 9(a). From the curves it is clear that the temperature differences along the core are negligible (in depth, the core can be considered at the same temperature) and above the fictive temperature. In the opposite, the cladding shows a significant temperature difference between the fiber's front surface (laser incidence) and its back surface (about 230K).

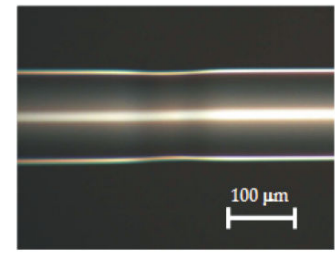

(a)

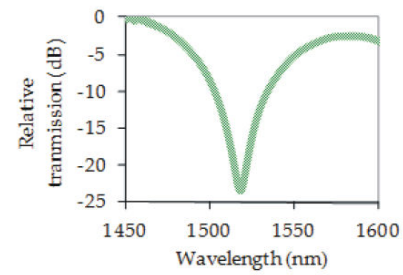

(b)

Figure 9. Picture showing (a) an irradiated zone belonging to a $25 \mathrm{~mm}$ LPFG with $500 \mu \mathrm{m}$ period and (b) respective relative transmission. (600 ms exposure time, 6 W laser power).

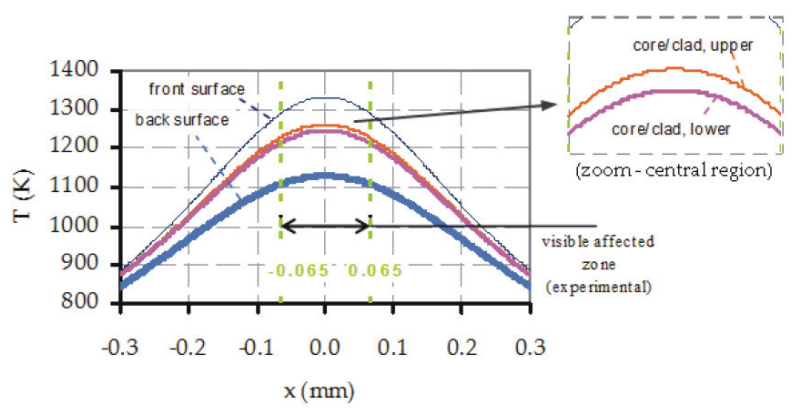

Figure 10. Temperature distribution at the fiber's axial direction at $t=0.6 \mathrm{~s}$. The curves were obtained at the optical fiber's front surface, core/cladding interfaces (upper and lower) and at the back surface of the fiber, and $x=y=0 \mathrm{~mm}$ (see Figure 8).

Using the set of equations (7), the residual axial elastic stresses in the cladding and core are approximately $0.05 \mathrm{MPa}$ (cladding) and $12.57 \mathrm{MPa}$ (core). Adding these values to the residual thermal stresses calculated using equation (6) the resulting residual stresses can be obtained. Figure 11(a) plots these values for $\mathrm{x}=0$ along the $\mathrm{z}$-axis. The asymmetry is clearly 
visible (mainly in the cladding). However, it has no significant impact in the refractive index profile (obtained by adding the refractive index change $\Delta \mathrm{n}$ to its initial value) resulting from the process as it can be observed in the plot in Figure 11(b).

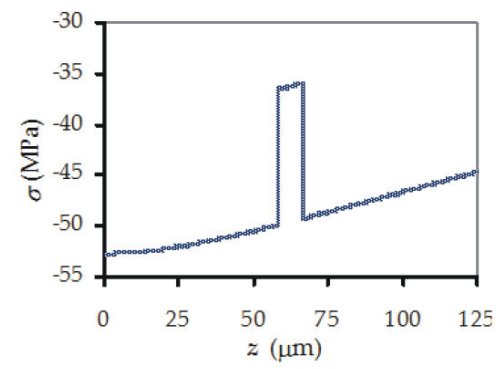

(a)

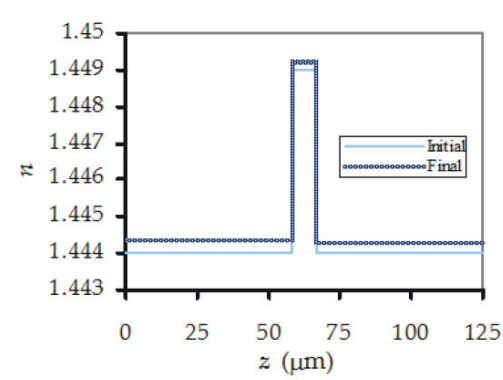

(b)

Figure 11. (a) Total residual stress and (b) refractive indexes (before and after laser irradiation) profiles, for the conditions considered.

Also evident is the imposing nature of the thermal component. However, if the drawing force increases, the balance between residual stresses changes. Figure 12 plots the refractive index change $\Delta n$ calculated for the core and cladding by increasing the weight. For lower weights, the core's refractive index increases while for weights higher than approximately $60 \mathrm{~g}$, it diminishes. At this value, the refractive index modulation is due mainly to the change in the cladding (which has almost no change with the weight value).

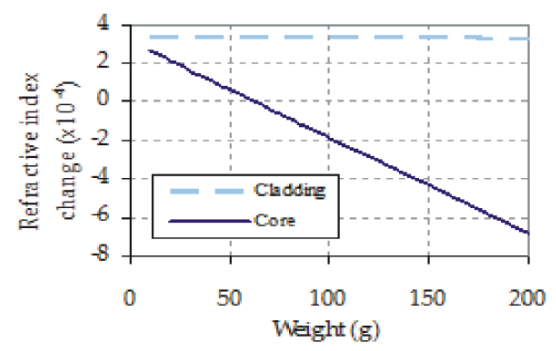

Figure 12. Refractive index change (core and cladding) with increasing weight, for the conditions considered.

\subsection{Multi-photonic NIR laser writing of FG sensors}

Besides single UV photonic absorption and MIR thermal effects, fs-pulse duration NIR (fsNIR) lasers appeared in the last years as alternative sources to write LPFG [7,50,51] and FBG $[50,52]$. In this case, the high peak power irradiation (typically in the order tenth's of thousands of $\mathrm{GW} / \mathrm{cm}^{2}$ ) produced by the fs-NIR laser induces high refractive index changes in the 
bulk glass material. This effect is considered as resulting from a non-linear multi-photonic absorption/ionization process in which material compaction and/or defect formation (depending on the intensity of the exposure) can occur [52]. Typically, $800 \mathrm{~nm}$ wavelength $\mathrm{Ti}^{3+}: \mathrm{Al}_{2} \mathrm{O}_{3}$ lasers are being used with pulses in the order of hundreds of fs. This laser makes use of the five-photon mechanism interaction with the silica-based optical fiber and $7.8 \mathrm{eV}$ band-gap energy for the common $3 \mathrm{~mol} \%$ Ge-doped fused silica core considered in the examples presented in this chapter [7].

Two types of writing procedures have been researched so far: one using a phase-mask process and the other a point-by-point writing. Both are similar to the techniques described previously for UV and MIR radiation writing. Thermo-stability (up to the glass transition temperature) of both laser written FBG and LPFG, and the ability of record in different types of fibers, as been reported as the main advantage of this technique. However, FBG fabricated using phase masks have strong cladding-mode absorption, only removed with careful relative positioning between the phase mask and the fiber, as well as with the choice of a special high order pitch phase mask [7]. High sensitivity to alignment is also reported [7,52] as one of the major drawbacks in fs-NIR technique regarding LPFGs, not only using masks but also in point-by-point writing. Nevertheless, the latter technique is being researched towards its application in the development of non-uniform (or "chirped") Bragg gratings [53] and direction-sensitive bending sensors [54].

\section{NIR laser micromachining for cavity-based sensors}

In recent years fiber micromachining has experienced an increasing development in the context of fiber sensing, the focus being made in creating intrinsic fiber optic structures, such as Fabry-Perot cavities, diffraction elements in the fiber end face, etc. To do so, the most traditional technique is based in the use of chemical etching. However, this technique (as others) is characterized by having low flexibility in its use. In the present, the preferred fabrication technique relies on laser etching, most notably fs or UV laser machining. This is a novel approach (basically following the principles already described in previous sections) being considered as having a huge potential, but the required equipment is complex and highly expensive. To overcome the present limitations the authors have been researching in applying ns-NIR pulses [20]. In this section this new technique is presented and its different applications illustrated. Based in the available experimental data, this optical fiber processing technique is analysed and its potential evaluated.

\subsection{Laser micropatterning}

Laser micropatterning refers to a material-removal process where micron-level features are fabricated in materials using a highly focused laser beam with high energy density, which is scanned over the material to create a specific feature. Ultra-fast lasers have pulse duration in the ns- through the fs-range which creates material removal by a vaporization process that 
limits material heating and allows materials to be micromachined with less dependence on laser wavelength absorption.

Micropatterning of hard materials, like glass, with pulsed lasers delivers the highest energy in the shortest possible time, thus reducing the material shock/impact effects. Applying laser energy over a relatively long time results in distortion of the microfeature, and other unwanted results, such as a large heat-affected zone, recast material, microcracking of the surface or inner walls or the laser beam not penetrating completely through the material thickness. These effects can be reduced by using a short $(<\mathrm{ms})$ pulse length.

One of the simplest ways to produce micro-patterns is to apply the concepts of laser drilling and appropriated scanning strategies. Traditional laser drilling techniques are: single pulse drilling, percussion (multiple pulses) drilling and trepanning. In this sequence, the required number of pulses increases, which can increase the machined volume.Basically, material removal in laser hole drilling relates with the vaporization of the material.

When dealing glass materials used in the development of fiber-based sensors, the laser interaction is conditioned by two important parameters: the wavelength and duration of the laser pulses. Since thermal impact can cause cracks in the glass after laser irradiation, UV radiation, having photon energies similar with those of glass, allows material removal by photonic processes without heating the material. Another possibility is to use ultra-short pulses $(<$ ps), so even in the NIR, photonic processes predominate over thermal effects. However, recent studies demonstrated that nanosecond pulses [14,20], in the NIR, can effectively be used to replace UV and fs-lasers in processing silica-based materials.

\subsection{Results on nanosecond NIR pulses micropatterning}

In 2011, Nespereira et al. [20] have presented the first results in creating micrometric holes in optical fibers using nanosecond NIR radiation. Since the tested optical fibers (standard communication silica-based fibers) have reduced absorption in the NIR (absorption coefficient around $1 \mathrm{~dB} / \mathrm{km}$ ) [47], the analysis made in section 4 regarding MIR interaction (with either core or cladding) cannot be made. So, although more research is needed (in particular to fully understand the physical principles involved), experiments allowed determining the conditions to vaporize the required amount of material. Holes with few microns and depths higher than $10 \mu \mathrm{m}$ were accomplished with multiple superposed shots. The analysis demonstrates the possibility of writing patterns and the potential in the development of fiber-based sensors.

\subsubsection{Experimental procedures}

Figure 13 illustrates the setup implemented and shows a picture of its implementation. Two main paths can be considered: an irradiation path, combining the laser source and an objective, and an observing path, were light reflected by the targeted fiber is observed by a CCD camera. Together with the fiber, a dichroic mirror is common to both paths allowing reflecting the emitted NIR laser beam, and transmitting visible light reflected by the fiber. 
The irradiation procedure was based on a pulsed Nd:YAG laser (BMI model: 5012 DNS 10c) operating at $1064 \mathrm{~nm}$ wavelength with a pulse width of $7 \mathrm{~ns}$ and $10 \mathrm{~Hz}$ repetition rate. The beam has a radius of $3.5 \mathrm{~mm}$ and is reflected by a dichroic mirror and focused into a SMF-28 optical fiber. The focusing optics is a 10x objective (ThorLabs LMH) with 0.25 numerical aperture, $20 \mathrm{~mm}$ effective focal length, designed to transmit high-power $1064 \mathrm{~nm}$ laser radiation and focus it to a diffraction-limited spot [20]. Thus, the spot radius on the fiber top is estimated to be about $3.7 \mu \mathrm{m}$. However, since the laser beam quality is low, having a $\mathrm{M}^{2}$ parameter higher than 2 (a Gaussian beam has $M^{2}=1$ ), the incident beam is expected to be focused into a $7.5 \mu \mathrm{m}$ spot radius $\left(M^{2} . w_{\mathrm{d}}\right)$.

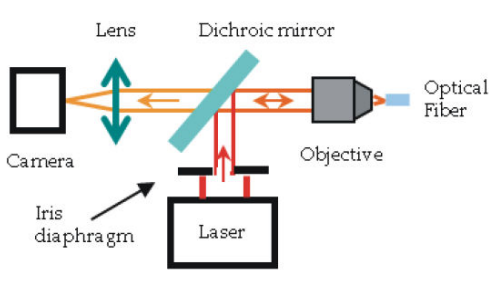

(a)

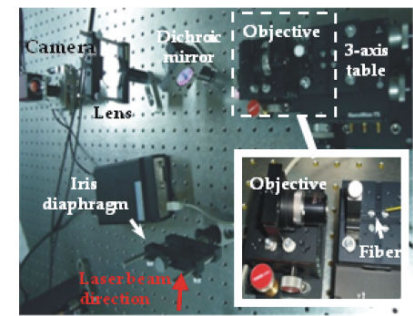

(b)

Figure 13. (a) Schematic and (b) photograph of the setup used for nanosecond pulsed NIR laser micropatterning of optical fibers.

Several operational parameters were considered. Besides changing the incident laser energy, the number of superposing pulses changed and it was also tested moving the fiber towards the focus after each pulse. Also tested was the impact of diminishing the spot size just at the laser's output, i.e. changing the depth of focus. This was accomplished with an iris diaphragm which allowed changing the beam from its initial $3.5 \mathrm{~mm}$ radius to about $2 \mathrm{~mm}$.

\subsubsection{Results and analysis}

Analysing the resulting data, tests [20] proved that single pulse drilling isn't effective in removing significant amount of material, especially when high depth is required. One laser pulse can produce a perfect round hole at the fiber'ssurface but with a depth less than $1 \mu \mathrm{m}$. However, increasing the number of superposing pulses lead effectively increased the hole's depth, while also increasing its diameter (Figure 14). As it can be seen, after about 8 pulses there isn't a significant change in the hole's diameter. However, its depth keeps increasing. More than 20 pulses damaged the fiber (cracks and breakage occurred).

The latter results were obtained with energy of $1.8 \mathrm{~mJ}$ and a $2 \mathrm{~mm}$ radius vignetted beam. Contrary to what could be expected the beam's size has low impact in the characteristics of the hole: its diameter only varies between $25 \mu \mathrm{m}$ and $31 \mu \mathrm{m}$, while the depth can be considered constant. However, the quality of the holes changes, being better for lower beam sizes as it can be seen in Figure 15 for the same energy and 10 laser pulses/hole. Also unexpected was the fact that increasing the laser energy, for a determined number of superposed pulses, 
or moving the fiber after each pulse, did not significantly alter the results. This can be a clear indication that some optical breakdown is the physical mechanism responsible by vaporising the material since once delivered enough energy to reach the breakdown threshold any further increase will not contribute for the process.

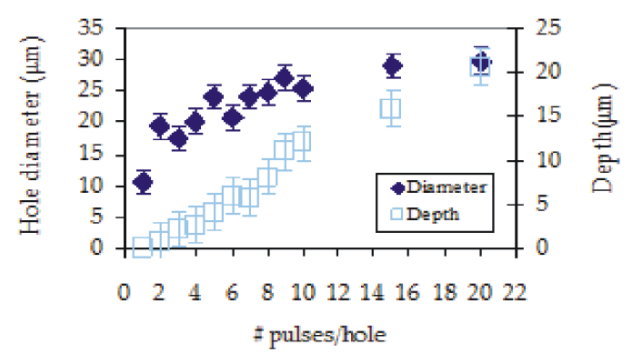

Figure 14. Measured hole's diameter and depth for different number of laser pulses per hole. (2 mm radius vignetted initial laser beam with $1.8 \mathrm{~mJ}$ incident energy).

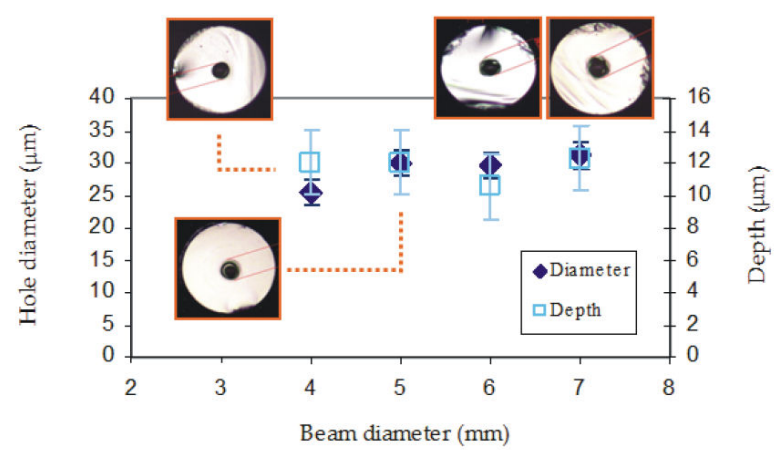

Figure 15. Measured hole's diameter and depth for different emitted laser beam diameter. Tests considered ten 1.8 $\mathrm{ml}$ laser pulses/hole.

These tests were made by irradiating the top of the fibers and the technique demonstrated that it is possible to obtain not only cavities for FP fiber sensors but also that different patterns can be inscribed (Figure 16(a)). Using the same parameters, it is also possible to microstructure the lateral side of the fiber. Figure 16, (b) and (c), shows the front and lateral views, taken by a microscope, of two holes opened in the side of a SMF-28 optical fiber.

Future work will focus in using nanosecond NIR pulses micropatterningto produce fiber sensors and also in studying and modelling the physical processes that rule the interaction phenomena. One possible alternative to the production of SPR sensors, while maintaining the same physical principle, is to replace the a posteriori metallization of the holes by direct 
formation of metallic nanoparticles simultaneously with the laser micropatterning of the fiber's top. This would require a metallic ion-doped fiber top. Nevertheless, some successful experiences were already made using NIR laser radiation, in the ns-pulse regime to obtain gold and copper nanoparticles in glass substrates [55,56]. Also, opening apertures along the fiber's length can lead to the development of new optical fiber sensors either by exposing the core or by giving access to inner hollow regions in photonic-crystal fibers.

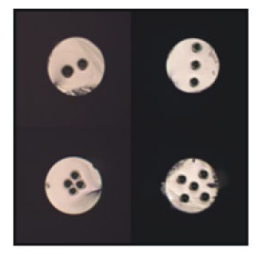

(a)

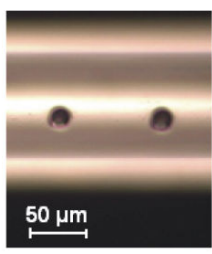

(b)

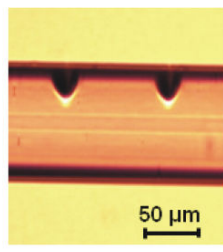

(c)

Figure 16. Examples of (a) different patterns written on the optical fiber's topand (b) front and (c) lateral views taken with an optical microscope for an example of two holes opened on the lateral side of a Corning SMF-28 fiber.

\section{Conclusions}

Laser technology plays an important role in the development of fiber-based optical sensors as its characteristics allow obtaining, in a controlled way, high quality features with good repeatability. Although some techniques are already well established, there still are many improvements and developments being researched. In particular, the use of IR radiation still presents challenges to overcome and promising new sensors are expected to be developed in a near future.

The use of MIR radiation in the writing of LPFG, namely through the use of $\mathrm{CO}_{2}$ laser systems, has proven to be an efficient tool. However, detail research in the study of the physical mechanisms involved in the process is still being done while its use to create new sensors is a parallel activity in photonic fields. As an engineering tool, a set of analytical expression were presented in this chapter which can give indications to the manufacturing process regarding the required operational parameter to accomplish a determined LPFG.

Recent advances in fs-pulses UV and NIR laser technology were described. In particular, inscribing FBG and LPFG is being researched, although some drawbacks are identified which limits its application. Besides that, an innovative technique that uses ns-NIR laser radiation to micropatterning optical fibers has been presented. These new results are challenging because the irradiated silica-based fibers are mainly transparent to NIR radiation and therefore the usual explanation based in direct heating by molecular or matrix vibrations induced by the laser beam (as in the previous section) should not hold. This leads to the necessity of a further in-depth analysis of the physical mechanisms involved. Nevertheless, the develop- 
ment of this technique opens new opportunities in the design of new cavity-based optical fiber sensors which are expected to appear in a near future.

\section{Acknowledgements}

This work was partially supported by the Portuguese Fundação para a Ciência e Tecnologia (FCT) through the project PTDC/FIS/119027/2010. The authors gratefully acknowledge José Luis Santos, Orlando Frazão, Pedro Jorge and Paulo Caldas from INESC-Porto for their advices and crucial contributions. A special thanks to Fernando Monteiro and António Oliveira for their technical support to the activities described in this paper.

\section{Author details}

João M. P. Coelho ${ }^{1,2}$, Marta Nespereira ${ }^{1}$, Catarina Silva ${ }^{1}$, Dionísio Pereira ${ }^{3}$ and José Rebordão ${ }^{1}$

1 Universidade de Lisboa, Faculdade de Ciências, Laboratório de Óptica, Lasers e Sistemas, Pólo do Lumiar, Estrada do Paço do Lumiar, Lisboa, Portugal

2 Universidade de Lisboa, Faculdade de Ciências, Instituto de Biofísica e Engenharia Biomédica, Campo Grande, Lisboa, Portugal

3 Nokia Siemens Networks, Rua Irmãos Siemens 1-1ª, Amadora, Portugal

\section{References}

[1] Dahotre NB, Harimkar SP. Laser Fabrication and Machining of Materials. New York: Springer; 2008.

[2] Neustruev VB. Colour centres in germanosilicate glass and optical fibres. Journal of Physics: Condensed Matter 1994; 6(35) 6901-6936.

[3] Kashyap R. Fiber Bragg Gratings. San Diego: Academic Press, 1999.

[4] Williams DL, Davey ST, Kashyap R, Armitage JR, Ainslie, BJ. Photosensitive germanosilicate preforms and fibers. In: Giancarlo C. Righini (ed.) Glasses for Optoelectronics II: proceedings of SPIE: 1513, 12 March 1991, The Hague, Netherlands. Bellingham: SPIE 1991.

[5] Grubsky V, Starodubov DS, Feinberg J. Photochemical Reaction of Hydrogen with Germanosilicate Glass Initiated by 3.4 5.4-eV Ultraviolet Light. Optics Letters 1999; 24(11): 729-731. 
[6] Mihailov SJ, Smelser CW, Lu P, Walker RB, Grobnic D, Ding H, Henderson G, Unruh J. Fiber Bragg Gratings Made with a Phase Mask and $800 \mathrm{~nm}$ Femtosecond Radiation. Optics Letters 2003; 28(12): 995-997.

[7] Nikogosyan D. Multi-photon High-excitation-energy Approach to Fiber Grating Inscription", Measurement, Science and Technology. 2007; 18 R1-R29.

[8] Smith AV, DoBT. Bulk and Surface Laser Damage of Silica by Picosecond and Nanosecond Pulses at $1064 \mathrm{~nm}$. Applied Optics 2008; 47(26): 4812-4832.

[9] Hill K, Fujii Y, Johnson D, Kawasaki B. Photosensitivity in optical fibre waveguides: application to reflection filter fabrication. Applied Physics. Letters 1978; 32(10): 647649.

[10] Erdogan T. Fiber grating spectra. Journal of Lightwave Technology 1997;15(8): 1277-1294.

[11] Lee D. Electromagnetic Principles of Integrated Optics. New York: John Willey and Sons; 1986.

[12] Othonos A. Fiber Bragg Gratings-fundamentals and Applications in Telecommunications and Sensing. Boston: Artech House; 1999.

[13] Jiang M, Zhang AP, Wang YC, Tam HY, He S. Fabrication of a Compact Reflective Long-period Grating Sensor with a Cladding-mode-selective

Fiber End-face Mirror. Optics Express 2009;17(20): 17976-17982.

[14] Vengsarkar A; Lemaire P; Judkins J, Bhatia V, Sipe J. Long-Period Fiber-GratingBased Gain Equalizers. Optics Letters 1996;21(5): 335-338.

[15] Erdogan T. Cladding-mode Resonances in Short- and Long-period Fiber Grating Filters. Journal of the Optical Society of America A 1997;14(8): 1760-1773.

[16] Costa RZV, Kamikawachi RC, Muller M, Fabris JL. Thermal characteristics of longperiod gratings $266 \mathrm{~nm}$ UV-point-by-point induced. Optics Communications 2009;282(5): 816-823.

[17] Patrick H, Chang C, Vohra S. Long Period Fiber Gratings for Structural Bending Sensing. Electronics Letters 1998;34: 1773 - 1775.

[18] Zhang Y, Chen X, Wang Y, Cooper K, Wang A. Microgap Multicavity Fabry-Pérot Biosensor. Journal of Lightwave Technology 2007; 25(7): 1797-1804.

[19] Lee CE, Gibler WN, Atkins RA, Taylor HF. In-Line Fiber Fabry-Pérot-Interferometer with High-Reflectance Internal Mirrors. Journal of Lightwave Technology 1992; 10(10): 1376-1379.

[20] Nespereira M, Silva C, Coelho J, Rebordão J. Nanosecond Laser Micropatterning of Optical Fibers. In: Costa M. (ed.) International Conference on Applications of Optics and Photonics: proceedings of SPIE on CD-ROM: 8001, 3-7 May 2011, Braga, Portugal. Bellingham: SPIE 2011. 
[21] Wei T, Han Y, Tsai HL, Xiao H. Miniaturized Fiber Inline Fabry-Perot Interferometer Fabricated with a Femtosecond Laser. Optics Letters 2008: 33(6): 536-538.

[22] Choi HY, Park KS, Park SJ, Paek UC, Lee BH, Choi ES. Miniature Fiber-optic High Temperature Sensor Based on a Hybrid Structured Fabry-Perot Interferometer. Optics Letters 2008; 33(21): 2455-2457.

[23] Rao YJ, Denga M, Duan DW, Zhu T. In-line Fiber Fabry-Perot Refractive-index Tip Sensor Based on Endlessly Photonic Crystal Fiber. Sensors and Actuators A: Physical 2008; 148(1): 33-38.

[24] Tafulo P, Frazão O, Jorge, PAS, Araújo FM. Intrinsic Fabry-Perot Cavity Sensor Based on Chemical Etching of a Multimode Graded Index Fiber Spliced to a Single Mode Fiber. In: Santos JL, Culshaw B, López-Higuera JM, MacPherson, WN. (eds.) EWOFS2010: Fourth European Workshop on Optical Fibre Sensors: Proceedings of SPIE: 7653, 8-10 September 2012, Porto, Portugal. Bellingham: SPIE 2010.

[25] Wei T, Han Y, Tsai H, Xiao H., Miniaturized Fiber Inline Fabry-Perot Interferometer Fabricated with a Femtosecond Laser. Optics Letters 2008; 33(6): 536538.

[26] Ran ZL, Rao YJ, Liu WJ, Liao X, Chiang KS. Laser-micromachined Fabry-Perot Optical Fiber Tip Sensor for High-resolution Temperature Independent Measurement of Refractive Index. Optics. Letters 2008; 16(3): 2252-2263.

[27] Kawasaki BS, Hill KO, Johnson DC, Fujii Y. Narrow-band Bragg reflectors in optical fibers. Optical Letters 1978; 3(2): 66-68.

[28] Vengsarkar AM, Lemaire PJ, Judkins JB, Bhatia B, Erdogan T, Sipe JE. Long-period fiber gratings as band-rejection filters. In Optical Fiber Communication Conference, OFC95, San Diego, CA, Feb. 1995, pp. PD4-2, 1995.

[29] Meltz G, Morey WW, Glenn WH. Formation of Bragg Gratings in Optical Fibers by a Transverse Holographic Method. Optics Letters 1989; 14(15): 823-825.

[30] Davis DD, Gaylord TK, Glytis EN, Kosinski SG, Mettler SC, Vengsarkar AM. Long Period Fibre Grating Fabrication with Focused CO2 Laser Pulses. Electronics Letter 1998;34(3): 302-303.

[31] Akiyama M, Nishide K, Shima K, Wada A, Yamauchi R. A Novel Long-period Fiber Grating Using Periodically Releases Residual Stress of Pure-silica Core Fiber. In: Optical Fiber Communication Conference (OFC), San José, CA, pp. 276-277, Feb. 1998. [Techn. Dig. Conf. Opt. Fiber Commun., 276, 1998].

[32] Wang Y. Review of Long Period Fiber Gratings Written by CO2 Laser. Journal of Applied Physics 2010; 108(8): 081101.

[33] Rego GM. Arc-induced Long-Period Fibre Gratings. Fabrication and their Application in Communications and Sensing. PhD thesis. University of Porto; 2006.

[34] Yablon AD. Optical and Mechanical Effects of Frozen-in Stresses and Strains in Optical Fibers. IEEE Journal of Selected Topics in Quantum Electronics 2004;10(2): 2004. 
[35] Yablon AD, Yan MF, Wisk P, DiMarcello FV, Fleming JW, Reed WA, Monberg EM, DiGiovanni DJ, Jasapara J. Refractive Index Perturbations in Optical Fibers Resulting from Frozen-in Viscoelasticity. Applied Physics Letters 2004;84(1):19-21.

[36] Yang S, Matthews M, Elhadj S, Draggoo V, Bisson S. Thermal Transport in CO2 Laser Irradiated Fused Silica: In Situ Measurements and Analysis. Journal if Applied Physics 1998;106(10): 103106.

[37] Grellier A, Zayer N, Pannell C. Heat Transfer Modelling in CO2 Laser Processing of Optical Fibres. Optics Communications 1998;152(4-6): 324-328.

[38] Coelho JMP, Abreu MA, Carvalho-Rodrigues F. Thermal Modeling CO2 Laser Radiation Transmission Welding of Superposed Thermoplastic Films. Optical Engineering 2003;42(11): 3365-3373.

[39] Coelho J, Abreu M, Carvalho-Rodrigues F. Modelling the Spot Shape Influence on High-speed Transmission Lap Welding of Thermoplastics Films. Optics, Lasers in Engineering 2008;46(1): 55-61.

[40] Coelho JMP, Nespereira MC, Abreu M, Rebordão JM. Modeling Refractive Index Change in Writing Long-period Fiber Gratings Using Mid-infrared Laser Radiation. In Proceedings of Summer AOP 2012 / X EONS, CD-ROM, June 27-29th, 2012, Porto, Portugal.

[41] Lancry M, Réginier E, Poumellec B. Fictive Temperature in Silica-based Glasses and its Application to Optical Fiber Manufacturing. Progress in Material Sciences 2012;57(1): 63-94.

[42] Mclachalan A, Meyer F. Temperature Dependence of the Extinction Coefficient of Fused Silica for CO2 Laser Wavelengths", Applied Optics 1987;26(9): 1728-1731.

[43] Xie J, Pan Q. Acousto-Optically Q-Switched CO2 Laser. In: Krzysztof Jakubczak (ed.) Laser Systems for Applications. Rijeka. InTech; 2012. p. 17-38.

[44] Rao YJ, Zhu T, Ran ZL, Wang YP, Jiang J, Hu AZ. Novel Long-period Fiber Gratings Written by High-frequency CO2 Laser Pulses and Applications in Optical Fiber Communication. Optics Communications 2004;229(1-6): 209-221.

[45] Oh ST, Han WT, Paek UC, Chung Y. Azimuthally Symmetric Long-period Fiber Gratings Fabricated with CO2 Laser. Microwave and Optical Technology Letters 2004;41(3): 188-190.

[46] Coelho J, Nespereira M, Silva C, Rebordão J. LOLS Research in Technology for the Development and Application of New Fiber-based Sensors. Sensors 2012;12(3): 2654-2666.

[47] Corning® SMF-28 optical fiber product information. New York: Corning Inc.: 2002.

[48] André P, Rocha A, Domingues F, Facão M. Thermal Effects in Optical Fibres. In: Marco Aurélio dos Santos Bernardes (ed.) Developments in Heat Transfer. Ridjeka: InTech; 2011. p1-20. 
[49] Clowes J, Syngellakis S, Zervas M. Pressure Sensitivity of Side-hole Optical Fiber Sensors. IEEE Photonics Technology Letters 2009;10(6): 857-859.

[50] Kondo Y, Nouchi K, Mitsuyu T, Watanabe M, Kazansky P, Hirao K. Fabrication of Long-period Fiber Gratings by Focused Irradiation of Infrared Femtosecond Laser Pulses. Optics Letters 1999;24(19): 646-648.

[51] Hindle F, Fertein E, Przygodzki C, Dürr F, Paccou L, Bocquet R, Niay P, Limberger H. Inscription of Long-period Gratings in Pure Silica and Germano-silicate Fiber Cores by Femtosecond Laser Irradiation. IEEE Photonics Technology Letters 2004; (16)8: 1861-1863.

[52] Mihailov SJ. Fiber Bragg Grating Sensors for Harsh Environments. Sensors 2012;12(2): 1898-1918.

[53] Fu H, Zhou K, Saffari P, Mou C, Zhang L, He S, Bennion I. Microchanneled Chirped Fiber Bragg Grating Formed by Femtosecond Laser-Aided Chemical Etching for Refractive Index and Temperature Measurements. IEEE Photonics Technology Letters 2008;20(19): 1609-1611.

[54] Martinez A, Lai Y, Dubov M, Khrushchev IY, Bennion I. Vector Bending Sensors Based on Fibre Bragg Gratings Inscribed by Infrared Femtosecond Laser. Electronics Letters 2005;41(8): 472-474.

[55] Silva C, Coelho JM, Ruivo A, de-Matos AP. Infrared Nanosecond Laser Effects on the Formation of Copper Nanoparticles. Materials Letters 2010;64(6): 705-707.

[56] Coelho JM, Silva C, Ruivo A, de-Matos A.P. Infrared Nanosecond Laser Radiation in the Creation of Gold and Copper Nanoparticles. In: Book of Abstracts, MATERIAIS 2011: VI International Materials Symposium, 18-20 April 2011 Guimarães: Portugal. Lisbon: SPM; 2011. 
